\title{
External Pulsed Plasma Propulsion And its Potential for the Near Future
}

\author{
J. A. Bonometti, P. J. Morton and G. R. Schmidt \\ NASA MSFC, TD40 \\ Marshall Space Flight Center, Alabama, 35812
}

Joe.Bonometti@msfc.nasa.gov/ (256) 544-4019 / Fax: (256) 544-5926

Phillip.Morton@msfc.nasa.gov/ (256) 544-4613 / Fax: (256) 544-5926

George.Schmidt@msfc.nasa.gov/ (256) 544-6055 / Fax: (256) 544-5926

\begin{abstract}
This paper examines External Pulsed Plasma Propulsion (EPPP), a propulsion concept that derives its thrust from plasma waves generated from a series of small, supercritical fission/fusion pulses behind an object in space. For spacecraft applications, a momentum transfer mechanism translates the intense plasma wave energy into a vehicle acceleration that is tolerable to the rest of the spacecraft and its crew. This propulsion concept offers extremely high performance in terms of both specific impulse (Isp) and thrust-to-weight ratio, something that other concepts based on available technology cannot do. The political concerns that suspended work on this type of system (i.e. termination of Project ORION) may now not be as insurmountable as they were in 1965. The appeal of EPPP stems from its relatively low cost and reusability, fast interplanetary transit times, safety and reliability, and independence from major technological breakthroughs. In fact, a first generation EPPP system based on modern-day technology (i.e., GABRIEL - an evolutionary framework of EPPP concepts) may very well be the only form of propulsion that could realistically be developed to perform ambitious human exploration beyond Mars in the 21 st century. It could also provide the most effective approach for deterrence against collision between earth and small planetary objects - a growing concern over recent years.
\end{abstract}

\section{INTRODUCTION}

NASA is currently conducting research on advanced propulsion technologies capable of supporting ambitious human exploration of the solar system in the early part of the next century. Most research to date has been geared towards concepts that offer tremendous performance improvements over current systems. The only problem is that virtually all of these technologies, such as fusion, antimatter and beamed-energy sails, have fundamental scientific issues and practical weaknesses that must be resolved before they can be seriously considered for actual applications. For instance, fusion is limited by the fact that we are still far away from demonstrating a device having energy gains sufficient for commercial power, let alone space applications. Antimatter has much appeal because of its high energy density, but it is severely hampered by extremely low propulsion efficiencies and high costs of current production methods. Beamed energy offers great potential too, but requires materials far beyond current state-of-the-art and tremendous investment in ground/space-based power beaming infrastructure.

Although we are optimistic that some of these issues will eventually be overcome, there is no guarantee that any of these technologies will be available by the first half of the next century. This state-of-affairs points to the disappointing fact that none of the advanced, high-power density propulsion concepts being considered by NASA. could, with any degree of certainty, meet the goals and timetables of NASA's own Strategic Plan. This is especially true in light of the conservative fiscal environment of the post-Apollo era, which could limit the sizable investment needed to resolve the fundamental issues associated with these concepts. Moreover, the cost for developing actual vehicles based on these technologies and their required infrastructure could realistically be on the order of hundreds of billions of dollars.

To obtain a quantum jump in propulsive capability by the early part of the next century, we must have safe, affordable systems with very high-power densities. Precedents suggest that any device engineered within the next 30 to 50 years should be based on the well-understood physics of today. The need for high power densities eliminates 
all but nuclear energy sources. The emphasis on known physics and affordability limits the scope still further to fission processes. Of the fission-based concepts that have been considered in the past (e.g., solid-core nuclear thermal, gas-core, internal and external nuclear pulse), only external nuclear pulse circumvents the Isp constraints imposed by containment of a heated gas, and provides the very high power densities needed for ambitious space transportation.

In the past, both internal and external pulse-engine concepts have been considered. Comparisons between these two approaches pointed to external pulse as the best candidate mainly because of its higher temperature limits and lower inert mass (Martin and Bond, 1979, Nance 1965). In addition, several researchers have investigated various forms of external momentum coupling. The most prominent examples are the standard pusher plate (Reynolds, 1972), the large lightweight sail/spinnaker (Solem, 1993), the rotating cable pusher (Cotter, 1971), and the combined pusher plate/magnetic field (Martin and Bond, 1979).

The most familiar effort in the area of external pulse-engines was Project ORION, which took place between 1958 and 1965. The Air Force spent approximately 8 million dollars on the program over its first 6 years (Prater, 1963). ORION, which was classified throughout most of its brief lifetime, engaged an impressive group of physicists and engineers who carried out numerous studies and tests on most aspects of the vehicle. The basic ORION design is shown in Figure 1. The proposed ships were large (from 10 to 30 meters in diameter) since performance tended to increase with diameter of the ship's pusher plate. This was due to the higher specific yields (i.e., burn up fractions) of larger pulse units, and the wider propellant interception angles at the minimum standoff distances allowed by material strength considerations. NASA funded several additional studies until 1965 when the entire effort was terminated - primarily for political reasons. The extensive analyses and experiments performed for ORION and subsequent studies indicate that spacecraft with high thrusts $(\sim 1$ to $10 \mathrm{~g}$ accelerations) and high Isp's $(\sim 10,000 \mathrm{sec})$ could be built, even with 1960's materials technology.
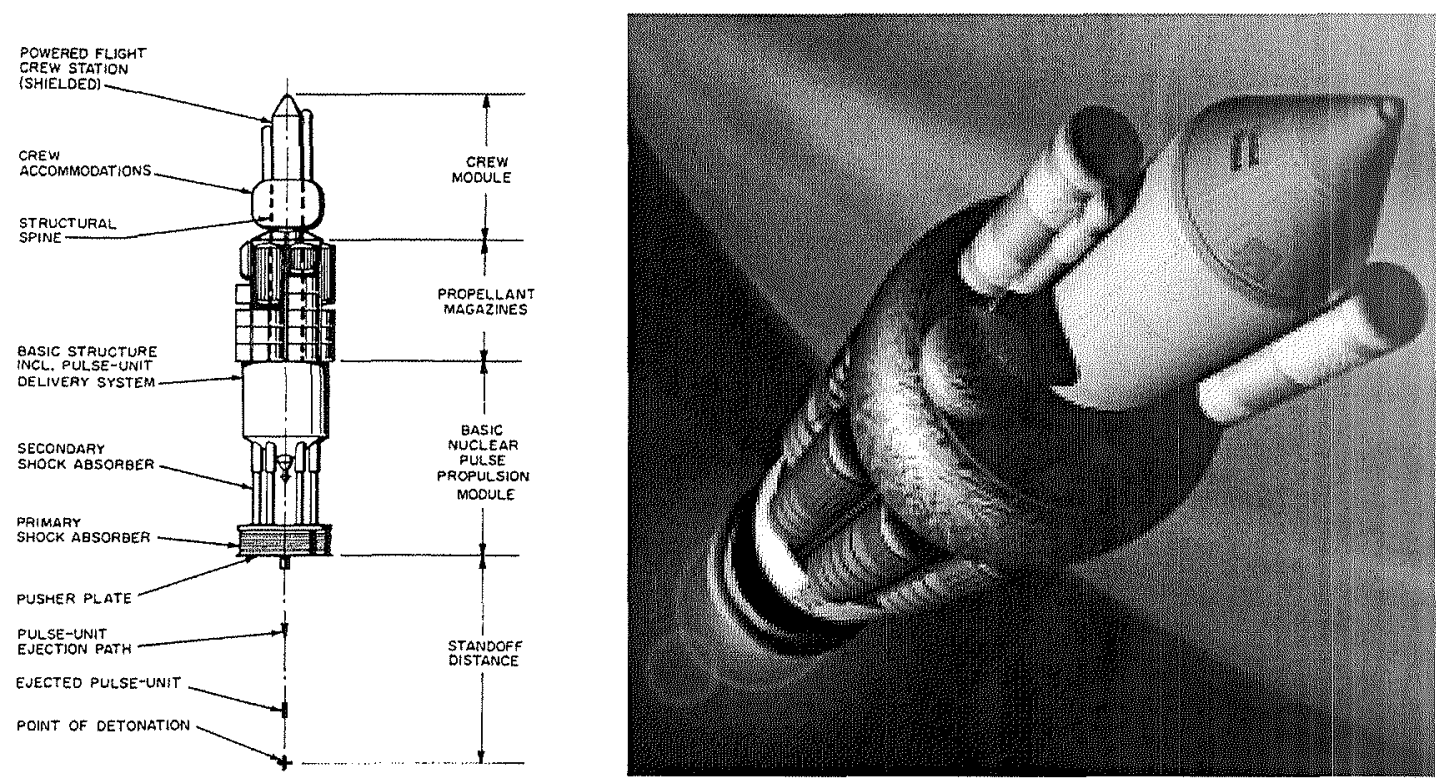

FIGURE 1. 1960 ORION concept.

\section{CONCEPT OVERVIEW}

At first glance, a nuclear pulse rocket appears to be quite radical, although it is conceptually very simple. Thrust is produced by ejecting and detonating small, fission-driven, pulse units at the aft end of the vehicle. This "external" engine operation, where the fission process is unconfined by material walls, is relatively independent of the reaction rate, temperature, pressure and other characteristics of the fuel. In practice, the system must be operated in a pulsed mode to allow the transfer of energy into a practical acceleration of the ship, which is limited by human and 
equipment tolerances. The physics behind creating a highly efficient fission burst is well understood, and in a vacuum, it produces a shell of ionized particles with an extremely high radial velocity. Thus, this concept of "riding on a plasma wave" is appropriately termed External Pulsed Plasma Propulsion or EPPP.

Key to EPPP's extraordinary performance are the facts that: (1) common materials can withstand an intense nuclear environment for very brief periods of time (i.e., nanoseconds), and (2) nuclear detonations are not only well understood, but also come much closer to achieving the maximum power density available from the fission process. Also, high thrust over a relatively short time imparts nearly optimum impulse to the vehicle for fast, efficient trajectories. In sharp contrast to the original ORION approach, recent analyses based on present-day considerations and technologies (e.g., dedicated in-space operation, low-energy pulse unit yields, low-ablation pusher plate materials) indicate that the performance advantages of EPPP could be applied to relatively small vehicles. If this is the case, then it is possible to develop small spacecraft that could carry human crews between Earth and Mars in just 1 to 3 months, as opposed to 6 to 12 months with chemical or nuclear thermal propulsion technology. In addition, EPPP would permit much more flexible return windows and eliminate the need for long stay times in the vicinity of Mars. Most importantly, EPPP provides a technology path leading to much higher Isp's ( 100,000 sec) using larger vehicles and more energetic detonations (e.g., fission/fusion and fusion) which could ultimately be used to open up the entire solar system to human exploration.

The main objection to EPPP has been the concern over nuclear contamination. Since modern-day practices would assuredly limit this concept strictly to space, radioactive contamination may not be as serious of issue as with ORION. Furthermore, the harsh environment of space has far more background radiation (particularly in the form of harmful gamma rays) than that produced by very small pulse units. Within 24 hours, the pulse unit's ionized mass dissipates completely into the background of the nominal space plasma density. Depending on the pulse unit efficiency, the exhaust velocities of the radioactive particles could exceed solar escape velocity (certainly beyond that of earth escape). Thus, there is no residue or permanent contamination to the environment beyond the natural sun's radiation.

\section{Application \#1: Human Interplanetary Exploration}

There are two reasons for seriously considering EPPP as an option for future development. The first is its potential for human exploration. Since the early years of the space program, most human exploration studies have concentrated on either the Moon or Mars. Although it is recognized in NASA's Strategic Vision that the ultimate goal is to extend human presence throughout the solar system and eventually the stars, only a negligible amount of effort has been devoted to these type of missions. EPPP provides a technology that would allow us to seriously consider missions to the outer planets. It would also enable dramatically shorter trip times to Mars and other nearer-term destinations.

The propulsion concepts that have been traditionally considered for Mars missions are chemical propulsion based on $\mathrm{O} 2 / \mathrm{H} 2$ combustion and solid-core nuclear thermal propulsion. Although the Isp of nuclear thermal $(-900 \mathrm{sec})$ is approximately twice that of chemical $(\sim 450 \mathrm{sec})$, both systems suffer from the same limitations with regards to trip time and mission planning. The main advantage of nuclear thermal is its potential to reduce vehicle mass in lowearth orbit, thus reducing the number of heavy-lift vehicle launches.

The performance that characterizes these two concepts favors Hohmann-type transfers into very slow heliocentric orbital trajectories. This narrows the available trajectories for return and necessitates long stays on the Mars surface while awaiting favorable return windows. This leaves the crew and equipment exposed to an extremely hostile environment for long periods of time - nominally 560 days surface stays with 170 to 200 day transit times (Kos, 1998). Cost is also significant, since earth launches are about half the mission budget in most conventional scenarios. Longer missions translate to larger payloads and more expendables, both of which increase launch requirements.

EPPP can solve this problem with its much higher Isp (5,000 to 10,000 seconds), while still providing the highthrust needed for fast orbit transfers. The result is higher energy transfer orbits, which could greatly reduce not only transit time, but permits broader return windows. This provides much more flexibility in mission planning and would not constrain the crew to long stay times on the Martian surface. It would also reduce the crew's exposure to the highly radioactive space environment and long periods of weightlessness. 


\section{Application \#2: Comet/Asteroid Deflection}

The other and perhaps most compelling application for EPPP is its use in asteroid or comet defense. Collisions between the Earth and small planetary objects occur frequently, with the typical result being that the objects burn up in the atmosphere. However, there is a low, but not negligible, probability of a collision with objects of sufficient size to cause catastrophic damage or an extinction-scale event. Good risk management would dictate that some effort be placed on devising countermeasures, if possible. Past studies identified a number of possibilities, almost all of which entailed ground and space-based infrastructure more extensive than that envisioned for ballistic missile defense. Because of the limitations of current propulsion technology, these systems would require permanent deployment of interceptors in deep space in order to allow engagement at a sufficient distance from Earth. In addition, the low-impulse methods of altering the object's trajectory, such as sails or electric thrusters, would probably not provide enough time for adequate trajectory alteration between detection and impact - especially in the case of a comet.

EPPP could be applied to the development of a much less expensive, purely ground-based deterrence system. If a likely catastrophic collision were identified, an EPPP-propelled interceptor could be launched into space using a conventional chemical launcher. It would have the power density necessary to rapidly travel to the target in time to force the threatening object from its collision course. The object's course change might be performed using sails or electric thrusters. However, these schemes are very risky since their effectiveness depends on the body's size, shape, speed, trajectory and many other properties. There is little room for error once the target is engaged, and the propulsion systems must operate reliably for very long durations to effect the change.

Alternatively, the same EPPP system that propelled the interceptor could be used to move the target. Single or successive pulse detonations at a predetermined distance from the asteroid's surface could be used to easily "nudge" the planetesimal and alter its course. The first wave of X-rays from the pulse would illuminate the planetesimal's surface causing ablation and thrust parallel to the object's projected area. The second wave of pulse fission products would produce another impulse in the same direction.

This approach has important advantages. It does not require asteroid capture or attachment of a propulsion unit to a highly variable surface. Since the "thrust" is parallel to the object's projected area, this approach is independent of the object's relatively indeterminate mass distribution and angular momentum. Also, the amount of impulse delivered can be easily tailored to any asteroid by the number of pulses, detonation standoff distance, and type of pulse unit.

\section{DESIGN CONCEPTS UNDER STUDY}

The realistic maximum Isp obtainable with fission-based EPPP is $\sim 100,000$ seconds. However, this type of performance would only be possible with very large spacecraft. Such vehicles would be impractical until the cost of access to space dropped substantially or in-space manufacturing became available. Therefore, a more conservative approach has been taken by considering smaller vehicles with lower performance (Isp $\leq 10,000$ seconds) using technology available in the near-term. This concept has been informally termed "GABRIEL." The GABRIEL series includes an evolutionary progression of vehicle concepts that build upon the nearest-term implementation of EPPP. This concept roadmap eventually culminates in larger systems that employ more sophisticated methods for pulse initiation and momentum transfer. GABRIEL is characterized by the following four levels:

1. Mark I: Solid pusher plate and conventional shock absorbers (small size)

2. Mark II: Electromagnetic coupling incorporated into the plate and shocks (medium size)

3. Mark III: Pusher plate extensions such as canopy, segments, cables (large size)

4. Mark IV: External pulse unit driver such as laser, antimatter, etc. (large size)

All of these levels, besides the GABRIEL Mark I, require technology that is not currently available, but may be attainable for a second-generation vehicle. The Mark I (Fig. 2) is also the smallest and least expensive version, but suffers from the poorest performance (nominally 5,000 seconds and 4 million newtons of thrust). Nonetheless, the Mark I has better Isp and thrust than any other known rocket system that could be reasonably developed within the next 20 years. Its heavy payload capacity and short trip times would significantly reduce the development challenges associated with manned spacecraft, as well as add extra safety margins through redundant systems, large reserve supplies and increased robustness. Interestingly, the same shielding used to protect the astronauts from solar 
flares could be used during engine operation (usually only a few hours at most), and the resulting radiation dose received would be much less than conventional multi-year missions. It is even conceivable that a vehicle with a performance as high as 4,000 seconds and 2 million newtons of thrust could be deployed and assembled in orbit using several Titan IV launch vehicles.

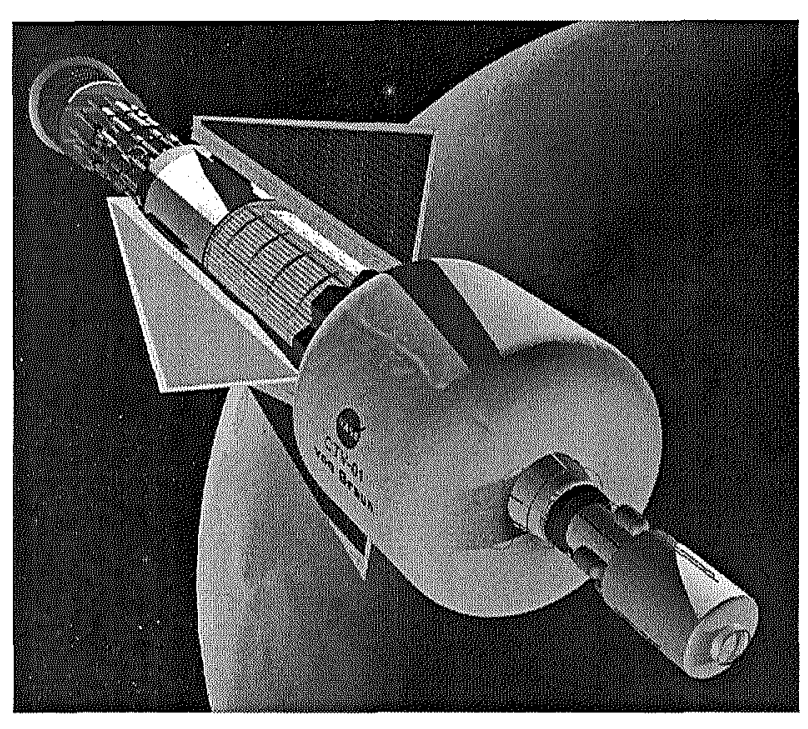

FIGURE 2. GABRIEL Mark I vehicle.

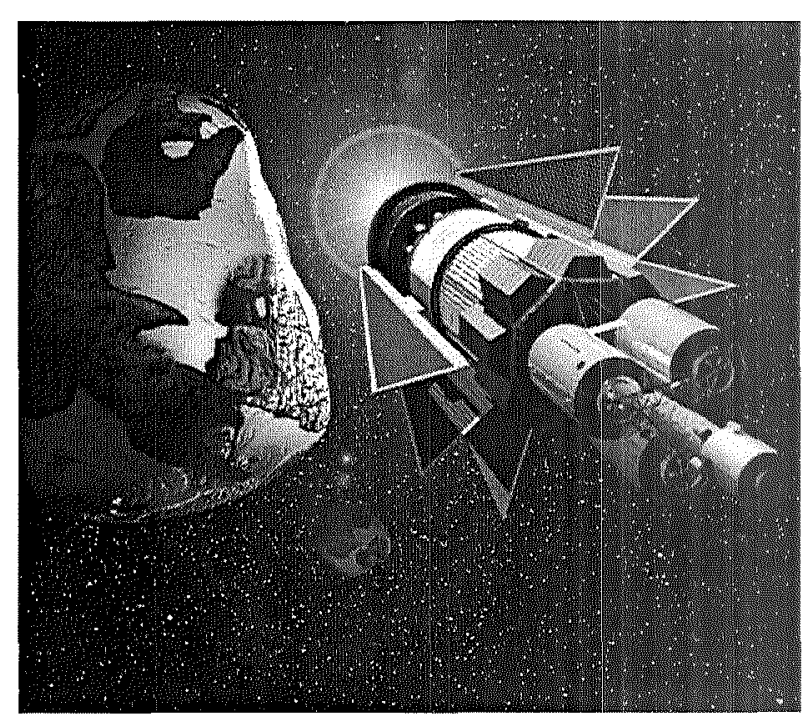

FIGURE 3. Asteroid deflection maneuver.

Several technical issues and trades must be addressed in order to define even a Mark I vehicle. These are the type of pulse unit, its degree of collimation, detonation position and fissile burn-up fraction. These issues dictate propulsion efficiency and drive design of the vehicle's mechanical elements. Another issue is the pusher plateplasma interaction. The amount of ablation experienced during each pulse could be significant and would dramatically affect Isp and thrust levels. Other issues include shock absorber efficiency, timing and dynamic response. Reusability will be important, so component wear must be kept to a minimum. In-space assembly, earth-to-orbit launch packaging and pulse unit safety and loading also must be addressed. Most of these issues have been investigated in the past and, although engineering challenges still remain, there are no formidable technical problems to overcome.

The ultimate hurdle in developing EPPP would be political in nature. Although GABRIEL does not face any insurmountable technical or financial obstacles, it does face one of perception. Use of nuclear material is almost always met by vehement opposition. However, there have been some important changes in the political landscape that may afford EPPP a chance where ORION failed. The Cold War is over and the fears of a large-scale nuclear conflict have abated somewhat. The existing ban on nuclear weapons in space actually has provisions that may allow peaceful uses of EPPP-type techniques below certain energies.

Even if EPPP is still viewed as too controversial for development in the near future, it would be worthwhile to begin reexamining it within the context of modern technologies and capabilities. Unlike physics, the sociopolitical environment does change, and a propulsion system with this tremendous capability may be needed - possibly on rather short notice (Fig. 3). The fact that many of the advanced propulsion concepts being researched now may never move beyond the "proof-of-principle" phase suggests that EPPP may be the only option we have for very ambitious human exploration of space in the foreseeable future.

\section{SUMMARY}

The case for reexamining nuclear pulsed propulsion and more modern embodiments of the EPPP concept has been made. The modern version of this propulsion concept, GABRIEL, is distinguished by its superior performance (i.e., both high Isp and high thrust-to-weight), its practicality (borrowing from only existing technologies), benign 
environmental impact (i.e., dedicated in space operation and reduced crew radiation exposure) and its economics (i.e., small size and reusability). More advanced systems with much better performance could be developed as technology in key areas mature. Improved performance can be achieved through advanced materials, magnetic fields (both on the pusher plate and along the shocks), novel momentum transfer schemes, and pulse unit drivers.

However, it is the rationale for considering EPPP that is most important. EPPP offers a highly effective method for deflecting comets or asteroids. Trips to and from Mars may be significantly shorter and safer than with conventional propulsion concepts. The flexibility of missions employing EPPP is enormous, allowing massive payloads, emergency return capability and routine transit from a reusable vehicle. Beyond Mars, missions to the asteroid belt, Jupiter and other planets are possible with the same basic system.

Timing for development of EPPP may also be better than during the days of ORION. In many ways, international cooperation is more prevalent, and could conceivably be extended to the peaceful application of unused nuclear material. Stockpiles of fissionable material can be permanently disposed of and environmental contamination is negligible if used outside the earth's magnetosphere. Finally, the human race is at the threshold of truly exploring, developing resources and permanently inhabiting space. GABRIEL may provide the best means of accomplishing this in the near future.

\section{ACKNOWLEDGMENTS}

The authors would like to thank George Dyson for providing much of the background documents on the ORION program, and Peggy for providing the many final proof readings and grammatical corrections.

\section{REFERENCES}

Cotter, T. P., Rotating Cable Pusher for Pulsed-Propulsion Space Vehicle, Los Alamos Scientific Laboratory paper, LA4666-MS UC-33, Propulsion Systems and Energy Conversion TID-4500, 1971.

Kos, L., Human Mars Mission: Transportation Assessment, AIAA-98-5118, 1998.

Martin, A. R., Bond, A., Nuclear Pulse Propulsion: A Historical Review of an Advanced Propulsion Concept, Journal of the British Interplanetary Society, Vol. 32, pp.283-310, 1979.

Nance, J. C., Nuclear Pulse Propulsion, IEEE Transactions on Nuclear Science, February, 1965.

Prater, Lt., F. A. Gross, Nuclear Impulse Propulsion, Project 3775, (ORION), USAF document, 1963.

Reynolds, T. W., Effective Specific Impulse of External Nuclear Pulse Propulsion Systems, NASA Technical Note, NASA TN D-6984, 1972.

Serber, R., The Los Alamos Primer, Berkley CA, University of California Press, 1992.

Solem, J. C., Medusa: Nuclear Explosive Propulsion for Interplanetary Travel, Journal of the British Interplanetary Society, Vol. 46, pp. 21-26,1993. 